\title{
ON SOME REGULARITY CONDITIONS OF BOREL MEASURES ON $\mathbb{R}$
}

\author{
F. BEN NASR \\ (Received 1 May 1996; revised 3 December 1996) \\ Communicated by P. G. Dodds
}

\begin{abstract}
The aim of this paper is to resolve Taylor's question concerning certain regularity conditions on a Borel measure. The proposed solution is given in the framework of Brown, Michon and Peyrière, and Olsen.

1991 Mathematics subject classification (Amer. Math. Soc.): primary 28A60; secondary 60G57. Keywords and phrases: Hausdorff dimension, packing dimension.
\end{abstract}

\section{Introduction}

Let $\left\{\mathscr{F}_{n}\right\}_{n \geq 1}$ be a sequence of finite partitions of $[0,1$ [ by intervals, semi-open to the right. These partitions need not be nested. If $x \in\left[0,1\left[, I_{n}(x)\right.\right.$ stands for the intervals of the family $\mathscr{F}_{n}$ which contains $x$. The length of an interval $J$ is denoted by $|J|$. We suppose that, for any $x \in\left[0,1\left[, \lim _{n \rightarrow \infty}\left|I_{n}(x)\right|=0\right.\right.$.

We consider two indices dim and Dim which are defined as Hausdorff and Tricot dimensions [7], but only considering coverings and packings by intervals in the family $\left\{\mathscr{F}_{n}\right\}_{n \geq 1}$.

A Borel probability measure $\mu$ is called regular uni-dimensional if

$$
\exists \alpha: \quad \lim _{n \rightarrow+\infty} \frac{\log \mu\left(I_{n}(x)\right)}{\log \left|I_{n}(x)\right|}=\alpha \quad \mu \text {-a.e. }
$$

For $q, t \in \mathbb{R}$, define

(C) 1997 Australian Mathematical Society 0263-6115/97\$A2.00+0.00 


$$
\begin{aligned}
& H_{\mu}(q, t)=\lim _{\delta \rightarrow 0} \inf \left\{\sum_{j}^{\prime} \mu\left(I_{j}\right)^{q+1}\left|I_{j}\right|^{-t}:\left[0,1\left[=\cup I_{j}, I_{j} \in \cup_{n} \mathscr{F}_{n},\left|I_{j}\right|<\delta\right\},\right.\right. \\
& P_{\mu}(q, t)=\lim _{\delta \rightarrow 0} \sup \left\{\sum_{j}^{\prime} \mu\left(I_{j}\right)^{q+1}\left|I_{j}\right|^{-t}:\left(I_{j}\right)_{j} \text { disjoint, } I_{j} \in \cup_{n} \mathscr{F}_{n},\left|I_{j}\right|<\delta\right\}, \\
& b_{\mu}(q)=\sup \left\{t \in \mathbb{R}: H_{\mu}(q, t)=0\right\}, \quad B_{\mu}(q)=\sup \left\{t \in \mathbb{R}: P_{\mu}(q, t)=0\right\},
\end{aligned}
$$

where $\Sigma^{\prime}$ is the sum over those $j$ with $\mu\left(I_{j}\right) \neq 0$. The detailed properties of the functions $b_{\mu}$ can be found in $[3,4,5]$, and detailed properties of the function $B_{\mu}$ can be found in [4].

For any function $f$, we consider the following Legendre transform of $f$ :

$$
f^{*}(x)=\inf _{y \in \mathbb{R}}(x(y+1)-f(y))
$$

If we put

$$
\Delta_{s}=\left\{x \in \left[0,1\left[: \lim _{n \rightarrow+\infty} \frac{\log \mu\left(I_{n}(x)\right)}{\log \left|I_{n}(x)\right|}=s\right\}\right.\right.
$$

then the theorems in [4] imply that

$$
\operatorname{Dim} \Delta_{s} \leq B_{\mu}^{*}(s) \quad \text { for } \quad a_{1}=\sup _{q>-1} \frac{B_{\mu}(q)}{q+1} \leq s \leq a_{2}=\inf _{q<-1} \frac{B_{\mu}(q)}{q+1}
$$

and the theorems in $[3,4,5]$ imply that

$$
\operatorname{dim} \Delta_{s} \leq b_{\mu}^{*}(s) \quad \text { for } \quad c_{1}=\sup _{q>-1} \frac{b_{\mu}(q)}{q+1} \leq s \leq c_{2}=\inf _{q<-1} \frac{b_{\mu}(q)}{q+1}
$$

The aim of this paper is to resolve the following open problem of Taylor [6]: Find a regular uni-dimensional $\mu$ such that $a_{1}<a_{2}, c_{1}<c_{2}$ but

$$
\operatorname{dim} \Delta_{s} \neq b_{\mu}^{*}(s), \quad \operatorname{Dim} \Delta_{s} \neq B_{\mu}^{*}(s), \quad \operatorname{dim} \Delta_{s}=\operatorname{Dim} \Delta_{s} \quad \text { for some } s
$$

Moreover the Borel measure $\mu$ which we propose satisfies the regularity condition suggested by Olsen [4]: $b_{\mu}(q)=B_{\mu}(q)$ for all $q$. 


\section{Example}

Let $\mathscr{A}$ be the set of finite words over the alphabet $\{0,1\}$. The concatenation, just denoted by juxtaposition, endows $\mathscr{A}$ with the structure of a semigroup. The empty word, which is the unit, is denoted by $\omega$. The set of words of length $n$ is denoted by $\mathscr{A}_{n}$. For every $j \in \mathscr{A}$, we denote by $N_{k}(j)$ the number of times the letter $k$ appears in the word $j$. Let $\mathscr{A} \cup \partial \mathscr{A}$ be the natural compactification of $\mathscr{A}$ ( $\partial \mathscr{A}$ is the set of infinite words). For any $j \in \mathscr{A}$, we define $C_{j}$ to be the cylinder formed by the elements of $\partial \mathscr{A}$ starting with $j$.

Take $\alpha, \beta \in \mathbb{R}$ such that $1 / 3<\alpha<\beta<1 / 2$. A cylinder $C_{j}$ of order $n\left(j \in \mathscr{A}_{n}\right)$ is called of $\alpha$-type (respectively $\beta$-type) if we have:

$$
\left.\left|N_{0}(j) / n-\alpha\right|<1 / n \quad \text { (respectively }\left|N_{0}(j) / n-\beta\right|<1 / n\right) .
$$

For any cylinder $C_{j}, j \in \mathscr{A}_{n}$, of $\alpha$-or $\beta$-type, we define:

$$
\widetilde{C}_{j}=\left\{C_{l}: l \in \mathscr{A}_{n+6}, C_{l} \subset C_{j} \text { and } C_{l} \text { is of the same type as } C_{j}\right\} .
$$

It is easy to check that

$$
\exists n_{0}: \quad \forall n \geq n_{0}, \quad \forall j \in \mathscr{A}_{n}, \quad \widetilde{C}_{j} \geq 2 .
$$

For each $k \in \mathbb{N}$ we select, in a random way, $2^{k+1}$ cylinders of order $n_{0}+6 k$. The selection is done in steps. In the first step, we select two cylinders of order $n_{0}, C_{j_{0}}$ and $C_{j_{0}^{\prime}}$ with $C_{j_{0}}$ of $\alpha$-type and $C_{j_{0}^{\prime}}$ of $\beta$-type. From the $n$th step to the $(n+1)$ st step, we choose two elements of $\widetilde{C}_{j}$ for every $C_{j}$ of the $n$th step.

Let $l_{0}, l_{1}, p_{0}$ and $p_{1}$ be a real numbers such that

$$
\begin{gathered}
0<l_{1}<l_{0}, \quad 0<p_{0}<p_{1}, \quad l_{0}+l_{1}=1, \quad p_{0}+p_{1}=1, \\
\frac{\beta \log \left(p_{0} / p_{1}\right)+\log p_{1}}{\beta \log \left(l_{0} / l_{1}\right)+\log l_{1}}<1 .
\end{gathered}
$$

We construct a sequence $\left\{\mathscr{F}_{n}=\left\{I_{j}\right\}_{j \in \mathscr{A}_{n}}\right\}_{n \geq 0}$ of finite partitions of $[0,1[$ in semiopen intervals in the following way. The first partition contains the unique interval $I_{\omega}=[0,1[$. We obtain the $(n+1)$ st partition from the $n$th one by cutting each interval $I_{j}, j \in \mathscr{A}_{n}$, into two intervals $\left\{I_{j k}\right\}_{k=0.1}$ such that:

$$
\left|I_{j k}\right|= \begin{cases}l_{k}\left|I_{j}\right| & \text { if } C_{j} \text { contains a selected cylinder, } \\ \left|I_{j}\right| / 2 & \text { otherwise. }\end{cases}
$$

Now define a measure $\mu$ in the following way. For $j \in \mathscr{A}$ and $k \in\{0,1\}$ let

$$
\mu\left(I_{j k}\right)= \begin{cases}p_{k} \mu\left(I_{j}\right) & \text { if } I_{j} \text { contains a selected interval, } \\ \mu\left(I_{j}\right) / 2 & \text { otherwise. }\end{cases}
$$


( $I_{j}$ is selected if $C_{j}$ is selected).

Then clearly $\mu$ is regular uni-dimensional of index 1 :

$$
\lim _{n \rightarrow \infty} \frac{\log \mu\left(I_{n}(x)\right)}{\log \left|I_{n}(x)\right|}=1 \quad \mu \text {-a. e. }
$$

Note that the measure $\mu$ is not quasi-Bernoulli, that is, there is no positive number $M$ such that, for any $j$ and $k$ in $\mathscr{A}$, we have

$$
M^{-1} \mu\left(I_{j}\right) \mu\left(I_{k}\right) \leq \mu\left(I_{j k}\right) \leq M \mu\left(I_{j}\right) \mu\left(I_{k}\right) .
$$

Consider the following quantities.

$$
\begin{gathered}
C_{n}(q, t)=\frac{1}{n} \log \sum_{j \in \mathscr{A}_{n}} \mu\left(I_{j}\right)^{q+1}\left|I_{j}\right|^{-t}, \\
C(q, t)=\lim _{n \rightarrow \infty} \sup C_{n}(q, t) \text { and } \varphi(q)=\sup \{t ; C(q, t)<0\} .
\end{gathered}
$$

It is easy to check that $\varphi$ is finite, strictly increasing on $\mathbb{R}$ and

$$
\varphi(0)=0, \quad \varphi(q) \leq q \quad \text { for all } q \in \mathbb{R}
$$

Since $C$ is a convex finite function, the function $\varphi$ is defined by the equality $C(x, \varphi(x))$ $=0$. We prove that

$$
b_{\mu}=B_{\mu}=\varphi \text {. }
$$

Property (3) results immediately from the following proposition.

PROPOSITION. For $q \in \mathbb{R}$,

(1) $\lim _{n \rightarrow \infty} C_{n}(q, \varphi(q))=0$ and $\lim _{n \rightarrow \infty} \inf n C_{n}(q, \varphi(q))>-\infty$.

(2) $b_{\mu}(q) \leq \varphi(q)$.

PrOOF. We introduce the following notation: for positive functions $u$ and $v, u \approx v$ means that there exists a positive constant $K$ such that $K^{-1} u \leq v \leq K u$.

Fix $q \in \mathbb{R}$ and put

$$
\begin{aligned}
A_{\alpha} & =\alpha\left((q+1) \log \frac{p_{0}}{p_{1}}-\varphi(q) \log \frac{l_{0}}{l_{1}}\right)+(q+1) \log p_{1}-\varphi(q) \log l_{1}, \\
A_{\beta} & =\beta\left((q+1) \log \frac{p_{0}}{p_{1}}-\varphi(q) \log \frac{l_{0}}{l_{1}}\right)+(q+1) \log p_{1}-\varphi(q) \log l_{1}, \\
\lambda & =2^{6(\varphi(q)-q)}, \quad \lambda_{\alpha}=2 e^{6 A_{\alpha}}, \quad \lambda_{\beta}=2 e^{6 A_{\beta}} .
\end{aligned}
$$


Let $Y_{n}$ denote the following mapping from $[0,1[$ to $\mathbb{R}$ :

$$
Y_{n}(x)=\mu\left(I_{n}(x)\right)^{q}\left|I_{n}(x)\right|^{-\varphi(q)} .
$$

Obviously, we have

$$
\int Y_{n} d \mu=e^{n C_{n}(q . \varphi(q))}
$$

For $k \in \mathbb{N}$, write $Z_{k}=Y_{n_{0}+6 k}$, and if $j \in \mathscr{A}$, define

$$
\begin{array}{r}
E_{k}^{p}(j)=\bigcup\left\{I_{i}: i \in \mathscr{A}_{n_{0}+6 k}, I_{i} \subset I_{j} \text { and } n_{0}+6 p\right. \text { is the largest order } \\
\text { of a selected interval containing } \left.I_{i}\right\} .
\end{array}
$$

Note that $E_{k}^{p}(j)$ could be reduced to the empty set.

\section{Proof of Assertion 1}

In order to establish the first assertion, we only need to prove

$$
\int Z_{k} d \mu \approx k \quad \text { or } \quad \int Z_{k} d \mu \approx 1 \text {. }
$$

We have

$$
\int Z_{k} d \mu=\int_{I_{j_{0}}} Z_{k} d \mu+\int_{I_{j_{0}^{\prime}}} Z_{k} d \mu+\int_{\bar{I}_{j_{0}} \cap \bar{I}_{j_{0}^{\prime}}} Z_{k} d \mu .
$$

It is easy to see that

$$
\int_{\bar{I}_{j_{0}} \cap \bar{l}_{j_{0}^{\prime}}^{\prime}} Z_{k} d \mu \approx \lambda^{k}
$$

On the other hand,

$$
\forall p \in \mathbb{N}, \quad 0 \leq p \leq k, \quad \int_{E_{k}^{p}\left(j_{0}\right)} Z_{k} d \mu \approx \lambda^{k-p} \lambda_{\alpha}^{p} .
$$

Since $\left\{E_{k}^{p}\left(j_{0}\right)\right\}_{0 \leq p \leq k}$ is a family covering $I_{j_{0}}$ whose elements are mutually disjoint, it follows that

$$
\int_{I_{j_{0}}} Z_{k} d \mu \approx \sum_{p=0}^{k} \lambda^{k-p} \lambda_{\alpha}^{p}
$$

In a similar way, we can show that

$$
\int_{l_{j_{0}}^{\prime}} Z_{k} d \mu \approx \sum_{p=0}^{k} \lambda^{k-p} \lambda_{\beta}^{p} .
$$

Since $C(q, \varphi(q))=0$, the relations (5), (6), (7) and (8) imply (4). 


\section{Proof of Assertion 2}

Let us define a family of functions from $\left[0,1\left[\right.\right.$ to $\mathbb{R}_{+}$in the following way:

$$
g_{t}=\sum_{k=0}^{\infty} e^{-6 k t} Z_{k}
$$

This allows us to define the family

$$
P_{t}=\frac{g_{t}}{\int g_{t} d \mu} \mu \quad(t>0)
$$

of probability measures on $[0,1[$.

Let $j \in \mathscr{A}_{n_{0}+6 n}$; then we have

$$
P_{t}\left(I_{j}\right)=\frac{1}{\int g_{t} d \mu}\left[\sum_{k=0}^{n-1} e^{-6 k t} \int_{I_{j}} Z_{k} d \mu+\sum_{k=n}^{\infty} e^{-6 k t} \int_{I_{j}} Z_{k} d \mu\right] .
$$

For a fixed $k \geq n$, in order to evaluate the integral $\int_{l_{j}} Z_{k} d \mu$, we need to distinguish three cases.

1st case $\left(I_{j}\right.$ is not selected).

$$
\int_{I_{j}} Z_{k} d \mu=\lambda^{k-n} \mu\left(I_{j}\right)^{q+1}\left|I_{j}\right|^{-\varphi(q)} .
$$

2nd case ( $I_{j}$ selected, $\left.I_{j} \subset I_{j_{0}}\right)$. We have

$$
\forall p \in \mathbb{N}, n \leq p \leq k, \int_{E_{k}^{p}(j)} Z_{k} d \mu \approx \lambda^{k-p} \lambda_{\alpha}^{p-n} \mu\left(I_{j}\right)^{q+1}\left|I_{j}\right|^{-\varphi(q)} .
$$

Since $I_{j}=\cup_{p=n}^{k} E_{k}^{p}(j)$ we get

$$
\int_{I_{j}} Z_{k} d \mu \approx\left[\sum_{p=n}^{k} \lambda^{k-p} \lambda_{\alpha}^{p-n}\right] \mu\left(I_{j}\right)^{q+1}\left|I_{j}\right|^{-\varphi(q)} .
$$

3rd case $\left(I_{j}\right.$ selected, $\left.I_{j} \subset I_{j_{0}^{\prime}}\right)$.

$$
\int_{I_{j}} Z_{k} d \mu \approx\left[\sum_{p=n}^{k} \lambda^{k-p} \lambda_{\beta}^{p-n}\right] \mu\left(I_{j}\right)^{q+1}\left|I_{j}\right|^{-\varphi(q)} .
$$

When $t$ goes to $0, P_{t}$ has at least a weak limit $\nu$. By using the first assertion and the relations (4), (9), (10), (11) and (12), this weak limit $v$ satisfies

$$
\nu\left(I_{j}\right) \leq K \mu\left(I_{j}\right)^{q+1}\left|I_{j}\right|^{-\varphi(q)}
$$


where $K$ is a constant which does not depend on $I_{j}$. Since the intervals of order $n_{0}+6 k, k \in \mathbb{N}$, allow us to construct $b_{\mu}$, we conclude that $b_{\mu}(q) \leq \varphi(q)$.

This concludes the proof.

Put $\quad s=\frac{\beta \log \left(p_{0} / p_{1}\right)+\log p_{1}}{\beta \log \left(l_{0} / l_{1}\right)+\log l_{1}}$

and observe that $\Delta_{s} \neq \phi, \Delta_{s} \subset I_{j_{0}^{\prime}}$. Then (1) and (2) imply that $a_{1}=c_{1}<a_{2}=$ $c_{2}$. On the other hand, due to the theorem in [1], it follows from (1) and (2) that $\operatorname{Dim} \Delta_{s}<B_{\mu}^{*}(s)$.

Now, let us consider the Borel probability measure $w$ on $\left[0,1\left[\right.\right.$ such that $w\left(I_{j}\right)=2^{-k}$ for each selected interval $I_{j}$ of order $n_{0}+6 k, I_{j} \subset I_{j_{0}^{\prime}}$. Then $w$ is concentrated on $\Delta_{s}$. By using Billingsley's theorem [2] for dim and the associated result [6] for Dim, we obtain $\operatorname{Dim} \Delta_{s}=\operatorname{dim} \Delta_{s}$.

\section{References}

[1] F. Ben Nasr, 'Calcul de dimensions de packing', Colloq. Math. 71 (1996), 137-148.

[2] P. Billingsley, Ergodic theory and information (Wiley, New York, 1965).

[3] G. Brown, G. Michon and J. Peyriere, 'On the multifractal analysis of measures', J. Statist. Phys. 66 (1992), 775-790.

[4] L. Olsen, 'A multifractal formalism', Adv. Math. 116 (1996), 82-196.

[5] J. Peyriere, 'Multifractal measures', in: NATO Adv. Study Inst. Ser. C 372 (Nijhoff, Dordrecht, 1992) pp. 175-186.

[6] S. J. Taylor, 'The fractal analysis of Borel measures in $\mathbb{R}^{d}$, J. Fourier Anal. Appl., to appear.

[7] C. Tricot, 'Two definitions of fractional dimension', Math. Proc. Cambridge Philos. Soc. 91 (1982), $57-74$.

Faculté des Sciences de Monastir

Département de Mathématiques

5000 Monastir

Tunisie 